\title{
Interface Currents: Supporting Fluent Collaboration on Tabletop Displays
}

\author{
Uta Hinrichs $^{1}$, Sheelagh Carpendale ${ }^{2}$, Stacey D. Scott ${ }^{2}$, and Eric Pattison ${ }^{2}$ \\ 1 Department of Computer Science, Otto-von-Guericke University, \\ Magdeburg, Germany \\ uta.hinrichs@student . uni-magdeburg.de \\ 2 Department of Computer Science, University of Calgary, \\ Calgary, Alberta, Canada \\ \{sheelagh, sdscott, ericp\}@cpsc.ucalgary.ca
}

\begin{abstract}
Large horizontal displays provide new opportunities to support individual and collaborative activities such as creativity and organizational tasks. We present Interface Currents, a fluid interaction technique designed to support face-to-face collaboration by improving access to and sharing of workspace items. Interface Currents are flexible containers that provide a controllable flow of interface items that support creativity during collaborative tasks and enable intuitive organization and sharing of digital information around horizontal displays.
\end{abstract}

\section{Introduction}

Many types of digital endeavours would benefit from more working space than is offered by common desktop displays. A single user trying to work with a large volume of materials or a group of users performing a planning, organizing, or brainstorming activity may benefit from a larger digital workspace. Highresolution, large-screen tabletop displays offer great potential for enabling such activities. Interactive tabletop displays have been developed to support activities such as information browsing 812]1314]15, photo sharing [12, and document sharing [16]. However, with the advantages of increased size, these large displays come hand in hand with new interfaces issues.

Large tabletop displays afford quite different interactions than common 21 inch desktop displays and, thus, introduce several issues for interface designers that vary considerably from those investigated in Xerox Star [6] derivative desktop interfaces. For example, workspace items may be out of reach because they are located on the opposite side of the table from a user's current position. Furthermore, reaching across the table can also have territoriality issues during collaboration [10. For instance, it is socially awkward to reach across another person's work or into that person's workspace [10. Thus, interfaces for tabletop displays must provide better access to workspace items for both individual and collaborative interactions. They should also support easy passing and sharing

A. Butz et al. (Eds.): SG 2005, LNCS 3638, pp. 185-197 2005.

(C) Springer-Verlag Berlin Heidelberg 2005 
of workspace content among multiple users. People may also move around the table or adjust their seating arrangements as others join and leave the group and to suit their task needs 1111. Thus, large-display interfaces need to support mobility at the display, and therefore should not be designed to assume fixed user locations.

Interfaces for tabletop displays need to provide better access to workspace items whether they are in easy or hard to reach regions for both individual and collaborative interactions. They should provide greater ease for spreading, passing and sharing of workspace content and they should address the need for personal mobility during the creative process. To address these problems, we introduce the Interface Current, an interface component that can support a fluid and flexible interaction between users. Currents can facilitate access to workspace items and more readily support creative activities for either individuals or groups.

\section{Related Work}

While the concept of an Interface Current has not previously been considered as a type of interface component, several existing interfaces can be discussed in terms of a current. Other mechanisms have a facility for sharing and passing on large displays or incorporating fluidity into their interfaces.

The Interactive Table [8] at the Museum of Modern Art used a physical Lazy Susan on a table to display information, similar in concept to the Lazy Susan shown in Figure 1(a), Sharing and discussing the information between the visitors was stimulated and simplified by the Lazy Susan. The Interactive Table shows a way to present information intuitively to multiple users. However, in that system, the Lazy Susan is a physical device. In contrast, the Personal Digital Historian (PDH) [2]12 tabletop interface provides a digital, circular workspace that can be rotated like a virtual Lazy Susan. Applying an Interface Current to a region-based interface component provides an easy mechanism for creating a virtual Lazy Susan. In contrast to the virtual Lazy Susan on the PDH, Interface Currents support flexible, adjustable boundaries.

The Café Table [15] has a "Living Memory Flow Zone" around the table edge. In this zone, information objects flow. The "Flow Zone" was designed to support opportunistic browsing, which allows users to watch objects as they move by and to select desired objects either by stopping the flow with a touch or removing the chosen objects. However, the "Flow Zone" on the Café Table is not repositionable nor are its boundaries adjustable, features that are both provided by Interface Currents.

Ståhl and Lundberg's Pond 14 tabletop interface presents data elements in a three-dimensional virtual pond, providing a new interaction metaphor for searching and browsing digital media. Media items related to a recent search query float to the surface of the Pond. Items which have not been interacted with for some time sink to the bottom of the Pond, gradually disappearing. This garbage collection also happens if the Pond becomes crowded. While the Pond does not allow users to directly control the properties of the floating behaviour, 
this behaviour is similar to an Interface Cur-rent in that it uses the notion of a flow direction (up and down) and a flow speed. Geißler [4] introduces a gesture technique called "throwing" for large displays that supports moving items across large distances in a natural way. However, studies have shown that this technique is too imprecise 1. Baudisch et al. 11] developed the "Drag-and-Pick" technique that augments the "Drag-and-Drop" technique for large displays. When a user drags an item, icons that are of a compatible type and located in the direction of the users' drag, produce copies of themselves called tip icons. These tip icons are connected to the source icon with a visual rubber band and move towards the dragged item to facilitate reach. On a shared, collaborative workspace, it could be confusing to have several crossing rubber bands invoked by multiple users.

Continuous series of images such as digital video data are often referred to as "streams". Several techniques have emerged that honour the fluid spatial/temporal quality of this data. One is to provide "fluid interaction" by creating a trail of video frames and by introducing some flexibility through the use of lens technology 9. Another approach to creating interaction for video data is to present the trail in $3 \mathrm{D}$, providing context for browsing [18.

\section{The Concept of an Interface Current}

Problems related to reach, mobility, and sharing also exist in traditional work and social environments. Physical devices have been developed to mitigate these problems. Consider the various "Lazy Susan"-type devices used in homes and restaurants for sharing and accessing food and other items on tabletops (Figure 1(a)p, the water canals and conveyer belts used in Sushi restaurants to distribute food to customers seated at various locations at the Sushi bar (Figure 1(b) , rotating shelves in kitchen cupboards to access items stored in the cupboard (Figure 1(c) , and the airport luggage carousels (Figure $1(\mathrm{~d})$. These devices facilitate handling large amounts of items, and reducing the effort involved in accessing hard to reach items. The tabletop Lazy Susan (Figure 1(a) also eases sharing items with others at a table and allows people to be located at various positions around the table. The concept of Interface Currents draws

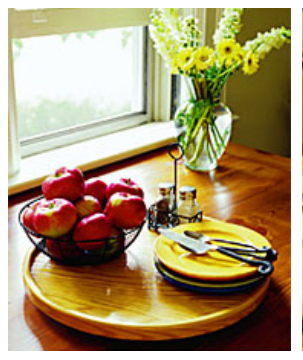

(a)

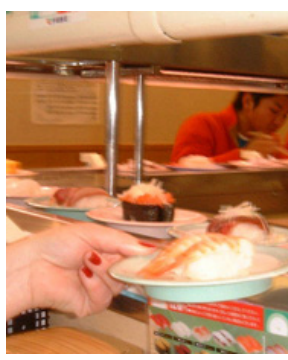

(b)

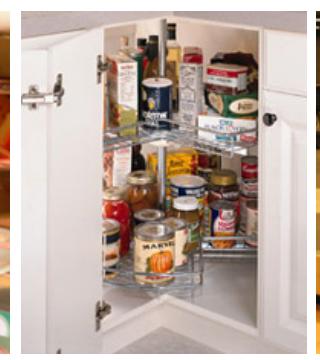

(c)

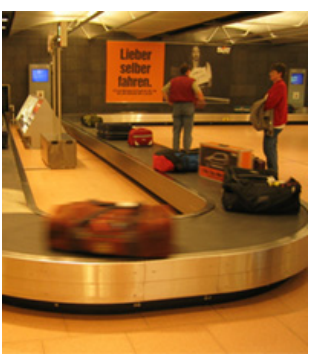

(d)

Fig. 1. Traditional Lazy Susans: (a) wooden tabletop Lazy Susan, (b) Sushi conveyer belt, (c) cupboard Lazy Susan, (d) luggage conveyer belt. 
on the physical solutions (Figure 1), as well as existing interface approaches, to provide a solution with additional flexibility.

A current is a continuous onward movement, traditionally thought of as existing in a body of liquid or gas. Characteristically, an Interface Current will move or run smoothly with unbroken continuity in a manner similar to a fluid. It has directional flow, speed, containment boundaries, and a location. Workspace items such as images, documents, or tools can be placed on a Current and be affected by its flow.

\subsection{Properties of an Interface Current}

The fundamental properties of an Interface Current are flow and the path in which this flow travels. Depending on usage, the Interface Current's visibility can also be changed.

Flow: Integral to the idea of a Current is its ability to flow. The flow has a specific direction and speed, which can be adjusted separately or in combination. In our prototype, this flow is invisible unless an item is placed on the Current.

Path: A Current flows in a path that has a location and some type of boundaries. The location of the path can either be fixed or mobile. The Current's boundaries define its shape and size, indicating areas in the workspace that are affected by its flow and areas that are not. In the extreme case the entire interface can be affected by a Current and the boundaries can be the edges of the workspace. Boundaries can be rigid in that they are established initially and not changed, or they can be flexible and controlled either by the system or by the user. A single external boundary creates a pool (Figure 2(a)) and an additional internal boundary makes a stream (Figure 2(b)) .

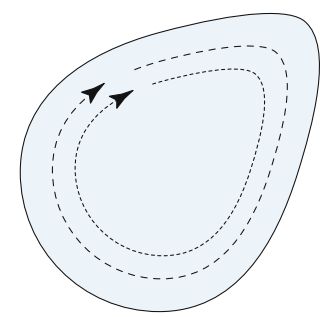

(a)

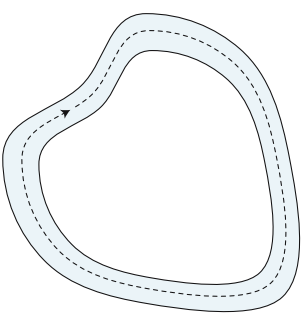

(b)

Fig. 2. Examples of Interface Currents: (a) pool with a Current and (b) stream

Visibility: While the path of an Interface Current determines its visual appearance, the location of the path affects its visibility. Depending on the function of the Current, its path can be totally visible, partly visible, or invisible. If an Interface Current is used as a personal storage area it can be reasonable to move it partly out of the workspace in order to save personal space (Figure 3). 
In contrast, Currents used in a group space for storing the results of a group brainstorming session, can be totally visible (e.g., the central Current shown in Figure 11(a)]. Any workspace items that are not currently needed can be moved out of the workspace (and retrieved when needed) by simply placing them on an Interface Current and moving the part or most of the Current out of the workspace.

\subsection{Purposes of an Interface Current}

Providing a Group Space: A group space is a shared work area on a tabletop display. Interface Currents in a group space can be used for organizing items that are accessible for all users at the display. Example group space tasks include brainstorming or design.

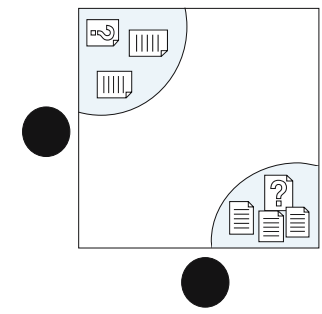

(a)

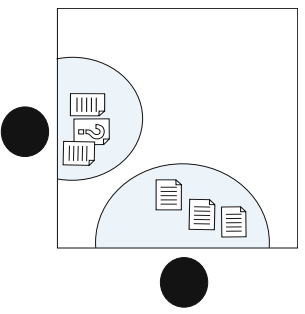

(b)

Fig. 3. Partly visible Interface Currents create more personal space

Providing Personal Storage Areas: Small or partly visible Interface Currents can be used for storing personal items. Partly visible Currents offer the opportunity to store large amounts of items, without crowding the personal workspace (Figure 31). The flow on the Current facilitates scrolling through the items in order to make invisible items visible and vice versa.

Facilitating Item Sharing: Interface Currents can be stretched out over a tabletop display in order to facilitate passing items. For instance, a Current can be used along the periphery of the tabletop workspace for passing content from one side of the table to the other (Figures 5(c) \& [11), similar to the sushi conveyor belt in Figure 1(b), A user can adjust the velocity of the flow of items to control the rate of item passing.

\subsection{Examples of Interface Currents}

To manifest a Current in an interface, decisions must be made about the Current's flow, path, boundaries, location, presentation, and the relationship it has to other inter-face components. In order to explore these issues, we have implemented two main types of Current-based interface components: Pools and Streams. User experiences with these two types of components have shown that each appears to support different types of interactions and task activities. 
Pools: The concept of a pool-shaped Current, or a Pool, comes most directly from the Lazy Susan-type devices (Figures 1(a) \& 1(c)). Rotating an entire workspace is an action common with traditional tabletop collaboration 12 and one that several existing tabletop interfaces have implemented 8 1213]17]. Pools are bordered by only one single exterior boundary. Our implementation of a Pool shows the area as a translucent colour and the boundary as an attenuated line, with several small control points along the boundary (Figure 4). Our default Pool boundary is a circle (Figure 4(a) . However, its boundary or circumference can be adjusted to almost any shape (e.g., Figure 4(b)]. A Pool can be expanded

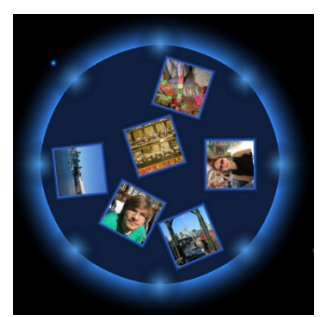

(a) Convex pool.

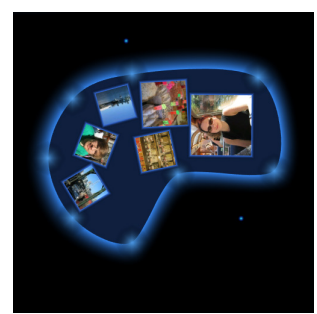

(b) Concave pool.

Fig. 4. Adjusting the boundary of a Pool

or shrunk; a user can pull a region towards themselves or push it out of their way if it interferes with their activities. The boundary control points can be used to adjust the boundary shape through the use of a Flow-type menu [5], invoked by touching the control point. The only indication of the Current's motion is the effect it has on the items placed on the Current. In order to solve the orientation problem on a tabletop display 7 all items are oriented towards the Pool's boundary. Once the flow on a Pool is started, items follow the shape of its boundary.

Streams: The concept of a stream-shaped Current, or a Stream, comes from streams of water. They are similar to the little "stream" in a sushi restaurant that carries various sushi selections to customers and to the airport luggage

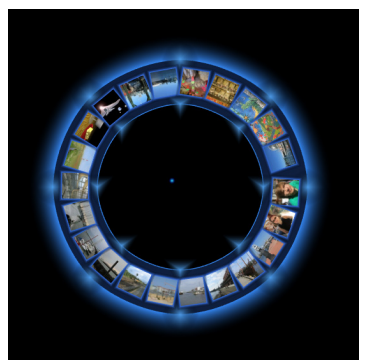

(a) Convex Current.

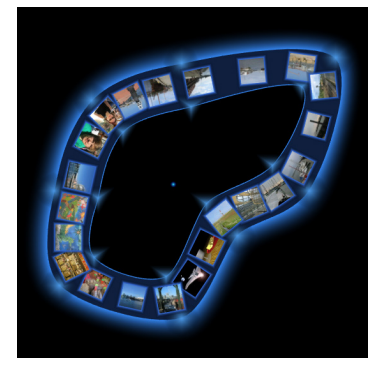

(b) Concave Current.

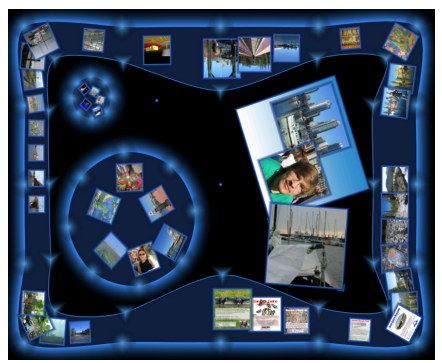

(c) Rectangular Current.

Fig. 5. Adjustable stream-shaped Currents 
conveyer belts (Figures 1(b) \& 1(d) . Our implementation shows a Stream as a ribbon of translucent color with an attenuated line along its outer boundary, a thin line along its inner boundary and small control points along each boundary (Figure 51). Streams can also be adjusted through a Flow-type menu [5] invoked via the boundary control points. They are adjustable to nearly any desired shape (e.g., Figure 5(b)p. Items on Streams move parallel to its boundaries. Similar to items contained in a Pool, they are automatically oriented its outer boundary.

\subsection{Interacting with Interface Currents}

Controlling the Appearance of the Current: To create a flexible, dynamically adjustable boundary we have used a basic four-point interpolating subdivision curve [3], as illustrated in Figure 6. All points generated by this method are on the boundary. We find the $\mathrm{C} 1$ continuity acceptable given that this method provides direct interactive control. We use eight (for peripheral Currents sixteen) initial points that are always visible in the interface, and five subdivision levels. Touching any of these points either by finger or by stylus, invokes a Flow-type menu [5] menu that offers different types of control over the Current' shape (e.g., Figures $7(\mathrm{a})$, 7(b) \& 7(c).

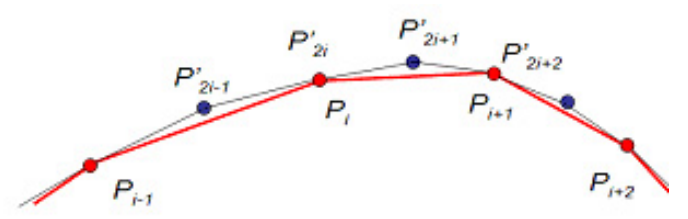

Fig. 6. Diagram of the 4-point interpolating subdivision algorithm

Adjusting the Boundary: Moving one's finger or a stylus while touching a menu icon in the Flow-type menu will change the geometric shape of the Interface Current. If it is a Pool, only the outer boundary changes: if it is a Stream, the path followed by both inner and outer boundaries changes (Figures 7(a) $\&$ 7(b) . With a Stream, however, it is also possible to manipulate the inner boundary alone in order to change the width of Stream at that location (Figure $7(\mathrm{c})$. Note that items on the Stream magnify or shrink depending on the Stream's width at that location. Thus, width of the Stream does not necessarily have to be constant along its entire flow path (Figure $7(\mathrm{c})$. In general, boundary adjustments allow any amount of stretching or compression of the Interface Current's size, no matter to which type of Current it is applied.

Manipulating the Currents' Position: An Interface Current can be moved to any location in the workspace, by simply touching and dragging on its outer boundary (Figures 7(d), 7(e) \& 7(f) . It can even be positioned partially outside the workspace and, thus, becoming partly invisible. Since easy access to the 
Current's flow still makes all items contained with the Current readily available, this expands useable space in a manner similar to use of Lazy Susans in kitchen cupboards (Figure 1(c)). As shown in Figure 5(c) it is possible to have multiple Interface Currents in the workspace. Through repositioning overlapping of different Currents can happen. In this case, floating items stay with the Currents to which they belong. There is no confusion of items moving from one Current to the next, unless that item is explicitly put there by a person.

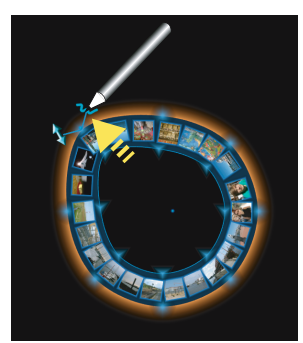

(a)

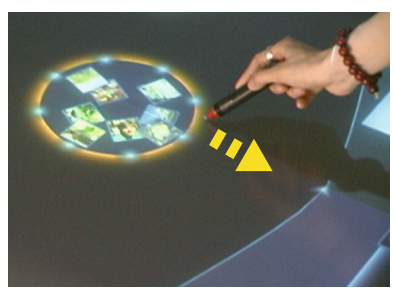

(d)

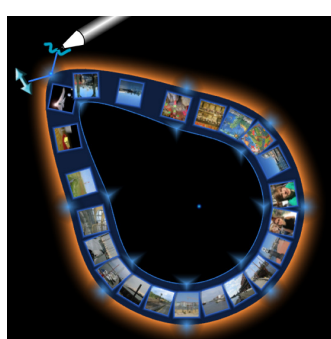

(b)

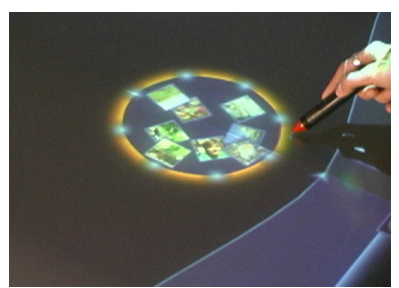

(e)

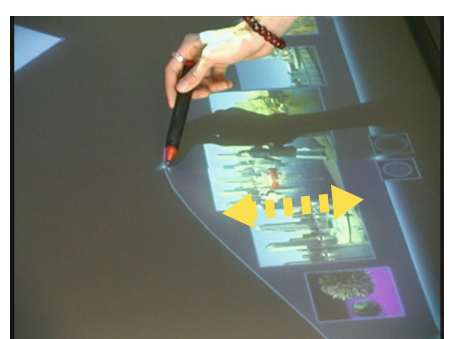

(c)

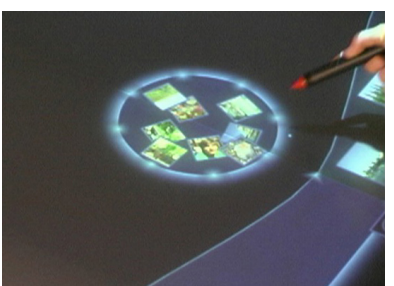

(f)

Fig. 7. Interaction with Currents: $(\mathrm{a}+\mathrm{b})$ adjusting a stream's shape both inner and outer boundary; (c) adjusting the inner boundary only; (d-f): relocating a Current.

Controlling the Flow on an Interface Current: By default, an Interface Current is floating continuously. In order to change the direction of the flow, a user simply has to indicate a new direction by touching inside the Current's boundaries (Figure 8(a)). Thus, if the items on the current are floating clockwise, moving the touch input counter-clockwise will reverse the direction of the flow. A brief touch on the Current will stop the flow and another contact with motion will start it again (Figures 8(b) \& 8(c) . The length of the gesture used to start or change the flow determines the speed of the flow. Our current implementation enables users to adjust the flow to three different speeds. The appropriate speed of the flow may depend on the task being performed.

Current-Item Interaction: An Interface Current does not need to contain any interface items. It can be simply an area in the workspace in which items can be placed for accessing, passing, and sharing. Workspace content, such as 


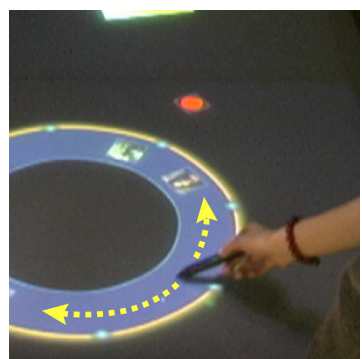

(a) Starting the flow.

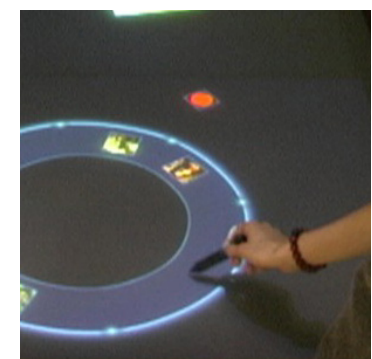

(b) Stopping the flow: Touching Current...

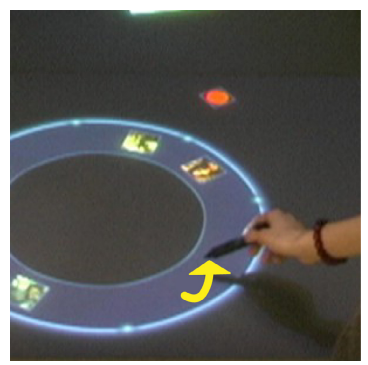

(c) ... and releasing stylus from Current quickly.

Fig. 8. Manipulating a Current's flow

images and text items, can be placed in it or removed from it. Figures 9(a) $\& 9(\mathrm{~b})$ show the addition of an image to a Pool. As soon as the image is placed on the Current, it is automatically resized to fit the size of the Current at that location. Content that is removed from a Current (see Figures 9(c) \& 9(d)) is returned to its original size.

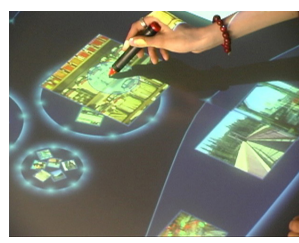

(a)

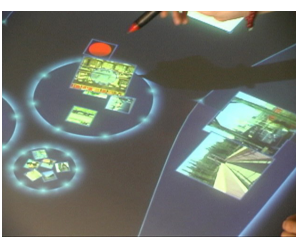

(b)

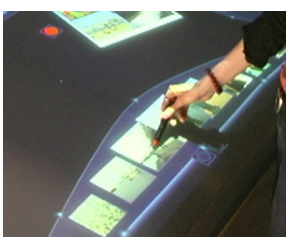

(c)

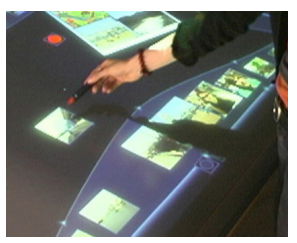

(d)

Fig. 9. Placing an item on a pool-based Current: (a) approaching the Current, (b) releasing the item onto the Current; (c-d) Removing an item from a Stream: (c) selecting the item in the Stream, (d) leaving the Stream.

Transforming Folders into Currents: To share content of folders easily between several people working on a horizontal display, folders can be transformed into Interface Currents. Double-clicking a folder in the workspace will evoke a folder-to-Current transformation (see Figures 10(a) \& 10(b)). This animated transformation results in a Stream-Current that includes the content of the folder spread out evenly and flow smoothly (see Figure 10(c)). The reverse transition can be activated changing a folder-generated Current back to a folder again. This can be very useful to save space in the workspace. The interaction with a Current created from a folder is the same as other Currents: The contents of a folder-Current can be adjusted and items can be added and removed. Adding and removing items will affect the contents of the associated folder.

When traditional folders are opened, their contents are arranged according to a single alignment. On a tabletop display, this single alignment of contents can 
make them difficult to view and interact with for those group members located at different sides of the table. Opening the folder to a Current can orient items towards the Current's outer edge, which is more appropriate when there are group members all around the table. Users could browse through the contents easily by activating the Current's flow.

\section{Discussion}

Interface Currents facilitate information sharing and access on tabletop displays. Through the combination of motion on demand and the high degree of flexibility, our Interface Current components support a large variety of tasks. Interface Currents can be used to ease access to items located at hard to reach areas of the workspace and provide a way of sharing information between people. For example, a Current can be stretched around the perimeter of a tabletop display so that a group of people can share access to a set of information (Figure 11) and be used for passing items back and forth. A Pool can be used to facilitate storing and sharing of items. Rotating a Pool using the Current's flow can bring far-away items closer, or it can be used to access workspace areas beyond the display space. Several Interface Currents can be used to spread information for brainstorming

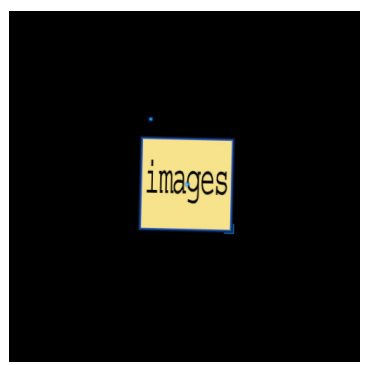

(a)

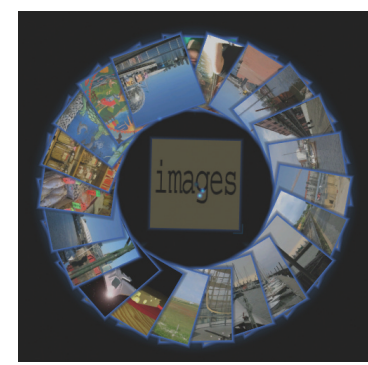

(b)

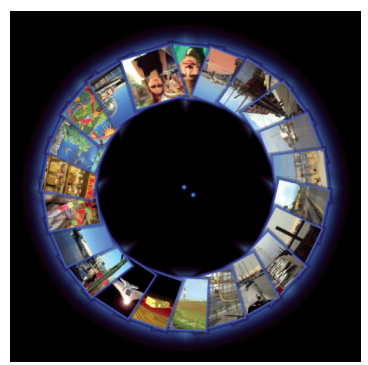

(c)

Fig. 10. Opening a folder into a stream-shaped Current: (a) the collapsed folder, (b) transition stage (folder fades and the Current opens), and (c) expanded folder.

activities (Figure 11). Currents can be shrunk and used for personal information access. The control of the Current's flow and the flexibility of its path help facilitate sharing. The flexibility of the paths allows people to tailor the Currents for the task at hand. For instance, a group of people who are collaborating can set up a Stream to exchange information by arranging the Current so that it passes conveniently near each of them. Figure 11 shows users working on a table with a Stream stretched around the edge of the tabletop workspace to operate like a conveyer belt. When one of these collaborators temporarily needs to attend to a different task, the Current can simply be pushed away to prevent interference. Currents lend themselves to being tucked out of the away, 


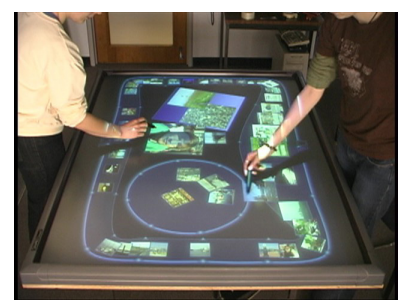

(a)

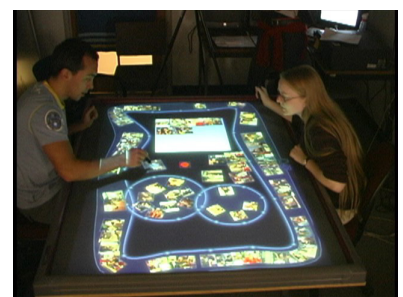

(b)

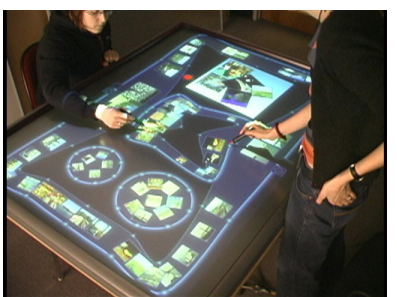

(c)

Fig. 11. A variety of uses of Interface Currents on a tabletop display

and by placing a considerable portion beyond the edge of the screen, extending the available screen space. A Current-based component may have two visible portions near opposite edges of the workspace and a connection between them that flows under the workspace. A user can place content on one of these visible portions and the current will carry the content under the workspace to appear on the other visible portion. This provides a transport or passing mechanism that does not interrupt work being performed in the main workspace area.

A user study exploring usage of and interaction with Interface Currents is underway. Preliminary results indicate that people with little computer experience found Interface Currents as supporting and intuitive to use, particularly for tasks that require creativity and the management of large amounts of information.

\section{Conclusion}

We have introduced the concept of Interface Currents to help address issues related to accessing and sharing of information at tabletop displays. A Current consists of a directional flow and a path in which this flow travels. We have presented several Current-based interface components, Pools and Streams which can be used to store, transport, and share workspace items. Current-based interface components such as Pools and Streams allow a user to manipulate workspace items indirectly, facilitating tasks such as reaching, passing, and sharing. Users can directly control the Current through easy mechanisms for starting, stopping, and changing the direction of the flow. We have designed these components with flexible boundaries that can be easily reshaped or repositioned to suit a users' immediate task needs. Currents introduce mobility into information access and organization. Mobility of workspace content is a step towards supporting the mobility of people using the interface. Workspace content can be readily moved as needed. Flexible boundaries support rapid and easy expansion, contraction, stretching and bending of regions that support information flow. Additionally, whole components such as Pools or Streams can be easily relocated. Interface Currents can be used to extend traditional interface components, such as folders, to improve the accessibility of these components on a tabletop display. Moreover, 
Interface Currents offer many interesting opportunities for developing new types of interface components to assist individual and collaborative interactions.

Acknowledgments. We would like to thank Natural Sciences and Engineering Research Council of Canada, Alberta's InformaticsCircle of Research Excellence, Alberta Ingenuity, and the Canadian Foundation of Innovation for research support. We also thank our fellow researchers from the Interactions Lab at the University of Calgary for their insightful comments on this work.

\section{References}

1. Baudisch, P., Cutrell, E., Robbins, D., Czerwinski, M., Tandler, P., Bederson, B., and Zierlinger, A.: Drag-and-Pop and Drag-and-Pick: Techniques for Accessing Remote Screen Content on Touch- and Pen-operated Systems. In Proceedings of Interact'03, 2003, pp. 57-64.

2. Dietz, P. and Leigh, D.: A Multi-User Touch Technology. In Proceedings of UIST'01 Symposium on User Interface Software and Technology, 4(4), 2001, pp. 219-226.

3. Dyn, N., Levin, D., and Gregory, J.: A Four-point Interpolatory Subdivision Scheme for Curve Design. Computer Aided Geometric Design, 4(4), 1987, pp. 257268.

4. Geißler, J.: Shuffle, Throw or Take it! Working efficiently with an Interactive Wall. CHI'98 conference summary on Human factors in computing systems, ACM, 1998, pp. 265-266.

5. Guimbretière, F., Winograd, T.: Combining Command Text and Parameter Entry. In Proceedings of UIST'00 Symposium on User Interface Software and Technology, ACM: NY, 2000, pp. 213-216.

6. Johnson, J., Roberts, T. L., Verplank, W., Smith, D. C., Irby, C., Beard, M., Mackey, K.: The Xerox Star: A retrospective. Computer, 22(9), 1989, pp. 11-29.

7. Kruger, R., Carpendale, M.S.T., Scott, S.D., Greenberg, S.: Roles of Orientation in Tabletop Collaboration: Comprehension, Coordination and Communication. In Journal of Computer Supported Collaborative Work, 13(5-6), 2004, pp. 501-537.

8. Omojola, O., Post, E.R., Hancher, M.D., Maguire, Y., Schoner, B.: An Installation of Interactive Furniture. In IBM Systems Journal,39 (3\&4), 2000, pp. 861-878.

9. Ramos, G., Balakrishnan, R. : Fluid Interaction Techniques for the Control and Annotation of Digital Video. In Proceedings of UIST'03 Symposium on User Interface Software and Technology, ACM: NY, 2003, pp. 105-114.

10. Scott, S.D., Carpendale, M.S.T, Inkpen, K.M.: Territoriality in Collaborative Tabletop Workspaces. In Proceedings of the ACM Conference on ComputerSupported Cooperative Work (CSCW)'04, 2004, pp. 294-303.

11. Scott, S.D., Grant, K.D., and Mandryk, R.L.: System Guidelines for Co-located, Collaborative Work on a Tabletop Display. In Proceedings of ECSCW'03 Conference on European Computer-Supported Cooperative Work, 2003, pp. 159-178.

12. Shen, C., Lesh, N., Moghaddam, B., Beardsley, P., and Bardsley, R.S.: Personal Digital Historian: User Interface Design. In Extended Abstracts of CHI '01 Conference on Human Factors in Computing Systems, ACM: NY, 2001, pp. 29-30.

13. Shen, C., Lesh, N., Vernier, F., Forlines, C., and Frost, J.: Building and Sharing Digital Group Histories. In Proceedings of CSCW'02 Conference on ComputerSupported Cooperative Work, ACM: NY, 2002, pp. 324-333. 
14. Ståhl, O., Wallberg, A., Soederberg, J., and Humble, J.: Information Exploration Using the Pond. In Proceedings of CVE'02 Conference on Collaborative Virtual Environments, ACM: NY,2002, pp. 72-79.

15. Stathis, K., de Bruijn, O., and Macedo, S.: Living Memory: Agent-Based Information Management for Connected Local Communities. Interacting with Computers,14(6),2002, pp. 663-688.

16. Streitz, N., Geißler, J., Holmer, T., Konomi, S., Müller-Tomfelde, C., Reischl, W., Rexroth, P., Seitz, P., and Steinmetz, R.: i-LAND: An Interactive Landscape for Creativity and Innovation. In Proceedings of CHI '99 Conference on Human Factors in Computing Systems, ACM: NY, 1999, pp. 120-127.

17. Vernier, F., Lesh, N., and Shen, C.: Visualization Techniques for Circular Tabletop Interfaces. In Proceedings of AVI '02. Conference on Advanced Visual Interfaces, ACM: NY, 2002, pp. 257-263.

18. Wittenburg, K., Forlines, C.: Rapid Serial Visual Presentation Techniques for Customer Digital Video Devices. In Proceedings of UIST'03 Symposium on User Interface Software and Technology, ACM: NY,2003, pp. 115-124. 